\title{
Evidence of New Intragenic HBB Haplotypes Model for the Prediction of Beta-Thalassemia in the Malaysian Population
}

Nur-Aisyah Aziz

Universiti Sultan Zainal Abidin

Wan-Rohani Wan Taib ( $\nabla$ wanrohani@unisza.edu.my)

Universiti Sultan Zainal Abidin

Nur-Khairunnisa Kharolazaman

Universiti Sultan Zainal Abidin

Imilia Ismail

Universiti Sultan Zainal Abidin

Hamid Ali Nagi Al-Jamal

Universiti Sultan Zainal Abidin

Wan-Arfah Nadiah Wan Abdul Jamil

Universiti Sultan Zainal Abidin

\section{Ezalia Esa}

Institute for Medical Research (IMR), National Institute for Health Malaysia

Hishamshah Ibrahim

Malaysia Thalassemia Registry, Medical Development Division, Ministry of Health $(\mathrm{MOH})$

\section{Research Article}

Keywords: Beta-thalassemia, HBB gene, haplotype, SNP, Malaysia

Posted Date: March 22nd, 2021

DOl: https://doi.org/10.21203/rs.3.rs-313477/v1

License: (c) (1) This work is licensed under a Creative Commons Attribution 4.0 International License.

Read Full License

Version of Record: A version of this preprint was published at Scientific Reports on August 18th, 2021. See the published version at https://doi.org/10.1038/s41598-021-96018-y. 
1 Evidence of new intragenic $H B B$ haplotypes model for the prediction of beta-

2 thalassemia in the Malaysian population

3

$4 \quad{ }^{1,2}$ Nur-Aisyah Aziz, ${ }^{1}$ Wan-Rohani Wan Taib, ${ }^{1}$ Nur-Khairunnisa Kharolazaman, ${ }^{1}$ Imilia 5 Ismail, ${ }^{1}$ Hamid Ali Nagi Al-Jamal, 'Wan-Arfah Nadiah Wan Abdul Jamil, ${ }^{2}$ Ezalia Esa, $6 \quad 3$ Hishamshah Ibrahim.

$7 \quad{ }^{1}$ Faculty of Health Sciences, Universiti Sultan Zainal Abidin, Gong Badak Campus, 8 Terengganu Malaysia.

9 2Molecular Genetics Laboratory, Haematology Unit, Cancer Research Centre, 10 Institute for Medical Research (IMR), National Institute of Health (NIH), Ministry of 11 Health Malaysia.

12 Malaysian Thalassemia Registry, Medical Development Division, Ministry of Health $13(\mathrm{MOH})$.

Corresponding author: Wan Rohani Wan Taib, Faculty of Health Sciences, Universiti 16 Sultan Zainal Abidin, Gong Badak Campus, Terengganu, Malaysia. Email: 17 wanrohani@unisza.edu.my 


\section{ABSTRACT}

This study sought to determine the potential role of $H B B$ haplotypes for the prediction of beta-thalassemia in the Malaysian population. A total of 543 archived samples were reviewed and selected for this study. Five tagging SNPs in the beta-globin gene $(H B B$; NG_000007.3) were recorded and analysed for SNP-based and haplotype association using SHEsis online software. Single-SNP-based association analysis showed three tagging SNPs have statistical significant association with beta-thalassemia; IVS2$16 G>C(p=0.036), I V S 2-666 C>T(p=0.032)$ and $3^{\prime} U T R+314 G>A(p=0.004)$. However, two tagging SNPs assigned as IVS2-74T>G and 3'UTR +233G>C did not yield significant association with p-value 0.099 and 0.211 , respectively. In a further investigation, combined five tagging SNPs for haplotype association analysis revealed three susceptible haplotypes with significant $p$-values of which, assigned as haplotypes 1-2-2-1-1 $\left(p=6.49 \times 10^{-7}, O R=10.371\right.$ [3.345 32.148]), 1-2-1-1-1 $(p=0.009$, $\mathrm{OR}=1.423[1.095 \sim 1.850]$ and $1-1-1-1-1 \quad\left(p=1.39 \times 10^{-4}, O R=10.221\right.$ [2.345 44.555]). Another three haplotypes were found to be protective haplotype with significant $p$ value of which assigned as haplotypes $2-2-1-1-1(p=0.006, O R=0.668$ [0.500 0.893]), 1-1-2-2-1 $(p=0.013, O R=0.357[0.153 \sim 0.830])$ and $1-1-2-1-1 \quad(p=0.033, O R=0.745$ [0.567 0.977]). This study has identified the potential use of intragenic polymorphic markers in the HBB gene, which were significantly associated with beta-thalassemia. A combination of these five tagging SNPs defined a new haplotype model for betathalassemia and further evaluation for the prediction of severity in beta-thalassemia.

Keyword: Beta-thalassemia, HBB gene, haplotype, SNP, Malaysia 


\section{INTRODUCTION}

Haemoglobin disorders are the most common monogenic disease worldwide ${ }^{1,2}$. These inherited autosomal recessive disorders are classified according to the haemoglobin expression or synthesis. There are three main categories of haemoglobin disorders; haemoglobinopathy is the structurally abnormal haemoglobin, thalassemia is the quantitatively reduced haemoglobin ${ }^{3,4}$ and, hereditary persistence of foetal haemoglobin (HPFH) and $\delta \beta$-thalassemia are characterized by increased levels of foetal haemoglobin $(\mathrm{HbF})$ in adulthood. The prevalence of haemoglobin disorders was high in tropical and subtropical regions such as sub-Saharan Africa, Mediterranean, Middle East, Indian subcontinent, and Southeast Asia ${ }^{3}$. However, due to modernization, people from epidemic areas migrated to the non-epidemic area. Hence, haemoglobin disorders have become a significant health problem in $71 \%$ of 229 countries globally 5 .

The recent report by the Malaysian Thalassemia Registry (MTR) has recorded a total of 8681 thalassemia cases from 2007 until November 2018. Since the launch of the National Thalassemia Preventive and Control Program in 2004, healthcare facilities have been upgraded to provide better quality for patient management. Hence, the survival rate of patients with thalassemia in Malaysia has improved ${ }^{6}$. With several molecular studies have been done previously in the East and West Malaysia, genetic heterogeneity is more observed in multiracial population in Malaysia with a diverse spectrum of alpha ( $\alpha-)$, beta $(\beta-)$ and delta $(\delta-)$ globin genes mutations among the patients with thalassemia syndromes ${ }^{7-11}$. Beta-thalassemia is due to decreased beta- 
globin chain synthesis of which, caused by a mutation in the HBB gene. The HBB gene mapped on chromosome 11p15.4 with a region spanning from 5,225,464 to 5,229,395 $b p$ on the reverse strand ${ }^{12}$. Therefore, the identification of nucleic acid variations in the HBB gene has improved our understanding of underlying causal mutations of betathalassemia in Malaysia.

The clinical presentation and molecular circumstantial of beta-thalassemia are highly heterogeneous and dependent on geographical and ethnicity factors ${ }^{13}$. The evidence may be derived from genetic predisposition, which is unique to particular ethnic groups, and that could enable targeted molecular analysis being designed [16, 17]. Genetic heterogeneity is more observed among different ethnicity in Malaysia with a diverse spectrum of HBB gene mutations. According to Elizabeth \&Ann (2010), approximately $73 \%$ of Malay patients with beta-thalassemia were due to mutation at codon $26(A>G)$ or HbE $\left(\beta^{E}\right)$, IVS1-5 $(G>C)$ (severe $\left.\beta^{+}\right)$and IVS1-1 $(G>T)\left(\beta^{\circ}\right)$. Whilst for Malaysian Chinese, $90 \%$ of beta-thalassemia cases were due to codon 41/42 (TTCT) $\left(\beta^{\circ}\right)$, IVS2-654(C>T), $-28(A>G)$, codon 17(A>T) and codon 71/72(+A). In East Malaysia, especially in Sabah, $90 \%$ of the Kadazan-Dusun with beta-thalassemia were due to $\beta$-Filipino deletion $\left(\beta^{\circ}\right)^{16,17}$.

However, the genetic variant interaction in conferring the effect based on haplotype inference has yet to be explored and refined in beta-thalassemia among the Malaysian population. Deciphering the predisposing effect by the potential haplotype markers can promote the exposition of underlying mechanisms of thalassemia development. In this study, five single nucleotide polymorphisms (SNPs) within the HBB gene were evaluated to determine its significance and haplotype structure inference with beta- 
thalassemia in Malaysia, which was the first study conducted in Malaysia to the best of our knowledge.

\section{RESULTS}

\section{Single association analysis}

In single-based association analysis, three tagging SNPs at IVS2-16G>C, IVS2666C $>$ T and 3'UTR $+314 G>$ A showed a statistically significant association with betathalassemia with $p$-value of $0.036, \quad O R=1.300$ [1.017 1.66]; $0.032, \quad O R=0.765$ [0.598 0.978] and 0.004, OR= 2.013 [1.238 3.272], respectively. However, tagging SNP at IVS2-74T>G and 3'UTR $+233 G>C$ did not show statistically significant association with beta-thalassemia of which, the $p$-value of 0.099 and 0.211 . The minor allele of these two variants showed a trend towards protective effect based on odds ratios less than $1 \quad(\mathrm{OR}=0.794 \quad[0.604 \sim 1.044]$ and $\mathrm{OR}=0.663$ [0.347 1.267] respectively). Table 1 depicts the genotypic association analysis of the five assigned SNPs. The most common genotype for IVS2-74T>G, IVS2-16G>C, IVS2-666C >T, 3'UTR $+233 G>C$ and $3^{\prime}$ UTR $+314 G>A$ was TT $(59.8 \%$ in case and $52.6 \%$ in control group), GC (49\% in case and $49.1 \%$ in control group), CT (43\% in case and $48.5 \%$ in control group), GG (94.4\% in case and $91.5 \%$ in control group) and GG (82.3\% in case and $90.4 \%$ in control group) respectively. Three tagging SNPs (IVS2-74T>G, 3'UTR $+233 \mathrm{G}>\mathrm{C}$ and 3 'UTR $+314 \mathrm{G}>\mathrm{A}$ ) showed high homozygosity rate in case and control groups. Meanwhile, a high heterozygosity rate was found in IVS2-16G>C and IVS2$666 \mathrm{C}>\mathrm{T}$ in both groups. 
Table 1: Single association analysis of five tagging SNPs of the HBB gene with beta-thalassemia

\begin{tabular}{|c|c|c|c|c|c|c|}
\hline \multirow{2}{*}{ SNP } & \multicolumn{3}{|c|}{ Genotype data (frequency) } & \multicolumn{3}{|c|}{ Case-control analysis } \\
\hline & $1 / 1$ & $1 / 2$ & $2 / 2$ & MAF & p-value & OR $[95 \% \mathrm{Cl}]$ \\
\hline \multirow[t]{2}{*}{ IVS2-74T>G } & $149(0.598)$ & $81(0.325)$ & $19(0.076)$ & $119(0.239)$ & 0.099 & 0.794 \\
\hline & $154(0.526)$ & $112(0.382)$ & $27(0.092)$ & $166(0.283)$ & & [0.604 1.044] \\
\hline \multirow[t]{2}{*}{ IVS2-16G>C } & $99(0.398)$ & 122(0.490) & $28(0.112)$ & $178(0.357)$ & 0.036 & 1.300 \\
\hline & $98(0.334)$ & $144(0.491)$ & $51(0.174)$ & $246(0.420)$ & & {$[1.017 \sim 1.66]$} \\
\hline \multirow[t]{2}{*}{ IVS2-666C>T } & $106(0.426)$ & $107(0.430)$ & $36(0.145)$ & $179(0.359)$ & 0.032 & 0.765 \\
\hline & $98(0.334)$ & 142(0.485) & $53(0.181)$ & $248(0.423)$ & & [0.598 0.978] \\
\hline \multirow[t]{2}{*}{ 3'UTR +233G>C } & 237(0.944) & $13(0.052)$ & $1(0.004)$ & $15(0.030)$ & 0.211 & 0.663 \\
\hline & 268(0.915) & $24(0.082)$ & $1(0.003)$ & $26(0.044)$ & & {$[0.347 \sim 1.267]$} \\
\hline \multirow[t]{2}{*}{ 3'UTR +314G>A } & 205(0.823) & $42(0.169)$ & $2(0.008)$ & $46(0.092$ & 0.004 & 2.013 \\
\hline & $263(0.904)$ & $28(0.096)$ & $0(0.000)$ & $28(0.048)$ & & [1.238 3.272] \\
\hline
\end{tabular}

Case data is at the top line, while control data is at the bottom line. The major allele is depicted as 1 . Minor allele is represented as 2. $M A F=$ Minor allele frequency. The $p$-value $<0.05$ is considered significant in Pearson Chi-Square. 


\section{Haplotype analysis}

Captivated by this favourable data in single association analysis, further investigation was conducted using combined allele from IVS2-74T>G, IVS2-16G>C, IVS2-666C>T, $3^{\prime}$ UTR $+233 G>C$ and $3^{\prime} U T R t+314 G>A$ of $H B B$ gene in an attempt to evaluate the predisposing effect of $H B B$ intragenic haplotype with beta-thalassemia. The naming system for the haplotype in this study is not related to the system used in the previous PCR-RFLP based haplotyping studies. Haplotype analysis revealed significant association for three haplotypes; 1-2-2-1-1, 1-2-1-1-1 and 1-1-1-1-1 with susceptibility effect towards beta-thalassemia of which, the $p$-values were $6.49 \times 10^{-7}(\mathrm{OR}=10.371$ $[3.345 \sim 32.148]), \quad 0.009\left(\mathrm{OR}=1.423 \quad[1.095 \sim 1.850]\right.$ and $1.39 \times 10^{-4} \quad(\mathrm{OR}=10.221$ [2.345 44.555]) respectively. On the other hand, three haplotypes; 2-2-1-1-1, 1-1-22-1 and 1-1-2-1-1 significantly conferred an opposing manner of effect to betathalassemia with the $p$-value 0.006 (OR=0.668 [0.500 0.893]), 0.013 (OR=0.357 [0.153 0.830]) and $0.033(\mathrm{OR}=0.745$ [0.567 0.977]) respectively. However, one haplotype with allele combinations 1-1-2-1-2 did not show any significant association with beta-thalassemia, where the $p$-value was 0.899 , yet the trend was towards susceptibility as depicted by $O R=1.041$ [0.559 1.939]). The summary of these findings was tabulated in Table 2. Haplotype with the frequency $<0.03$ in both controls and cases were automatically excluded from the analysis by the SHEsis online software. 
Table 2: Haplotype analysis of IVS2-74T>G, IVS2-16G>C, IVS2-666C>T, 3'UTR +233G>C and 3'UTR +314G>A in all races dataset with 249 cases and 294 controls

\begin{tabular}{|c|c|c|c|c|}
\hline **Haplotype & ${ }^{\star}$ Case (frequency) & ${ }^{\star}$ Control (frequency) & p-value & OR $[95 \% \mathrm{Cl}]$ \\
\hline $2-2-1-1-1$ & $95.08(0.192)$ & $164.67(0.284)$ & 0.006 & $0.668[0.500 \sim 0.893]$ \\
\hline $1-2-2-1-1$ & $26.38(0.053)$ & $3.43(0.006)$ & $6.49 \times 10^{-7}$ & $10.371[3.345 \sim 32.148]$ \\
\hline $1-2-1-1-1$ & $167.60(0.338)$ & $169.25(0.292)$ & 0.009 & $1.423[1.095 \sim 1.850]$ \\
\hline $1-1-2-2-1$ & $7.08(0.014)$ & $24.70(0.043)$ & 0.013 & $0.357[0.153 \sim 0.830]$ \\
\hline $1-1-2-1-2$ & $18.79(0.038)$ & $23.11(0.040)$ & 0.899 & 1.041 [0.559 1.939] \\
\hline $1-1-2-1-1$ & $120.08(0.242)$ & $188.91(0.326)$ & 0.033 & $0.745[0.567 \sim 0.977]$ \\
\hline 1-1-1-1-1 & $15.61(0.031)$ & $2.02(0.003)$ & $1.39 \times 10^{-4}$ & $10.221[2.345 \sim 44.555]$ \\
\hline
\end{tabular}

${ }^{\star}$ Frequency $<0.03$ in both controls and cases has been dropped in the analysis

**Major allele is depicted as 1. Minor allele is depicted as 2. A sequential in allele combination represents for IVS2-74T>G, IVS2-16G>C, IVS2-666C >T, 3'UTR +233G >C and 3'UTR +314G>A respectively 


\section{DISCUSSION}

In this study, we explored a single-based and haplotype association of five intragenic HBB polymorphisms in beta-thalassemia cases from Malaysia. It was suggested that the association of intragenic SNPs might be useful for the diagnosis and delineation of the clinical heterogeneity of beta-thalassemia ${ }^{19}$. Furthermore, the intragenic SNPs could be useful marker for linkage analysis and in prenatal diagnosis it can improve the diagnostic errors of which, caused by recombination ${ }^{20}$.

From the analysis of single-based association, two intronic polymorphisms; IVS2$16 \mathrm{G}>\mathrm{C}$, and IVS2-666C $>\mathrm{T}$, and one variant at $3^{\prime}$ untranslated region to $H B B$ gene assigned as 3'UTR $+314 \mathrm{G}>\mathrm{A}$ were found significantly associated with betathalassemia. The substitution of $C$ to $T$ allele at position 666 of intron 2 with minor allele frequency (MAF) of 0.359 in case group and 0.423 in control group conferring protection in beta-thalassemia with the odds ratio of $0.765(p=0.032)$. However, we noted that the MAF for IVS2-666C > T from this study was higher when compared with global MAF in the ClinVar (0.286) database but lower compared to 1000 Genome Project $(0.713)^{21}$. The genotype distribution of this intronic polymorphism revealed the heterozygote had yielded the highest frequency (48.5\%) in the control group. Association study done by Akhavan-Niaki et al. (2011) reported that IVS2-666C>T was found to be linked to a mutation at codon 8(-AA) [HBB:c.25_26delAA], of which this $\beta^{\circ}$-mutation was mainly described among the population from the Middle East and the Mediterranean. Hence, the authors suggested that IVS2-666C>T would be useful as a marker for codon 8 genotyping in prenatal diagnosis ${ }^{20}$. 
Meanwhile, two other variants showed a significant susceptibility effect towards betathalassemia: IVS2-16G>C and 3'UTR +314G>A. The MAF for IVS2-16G>C was 0.357 in the case group and 0.420 in the control group conferring susceptibility in betathalassemia with the odds ratio of $1.300(p=0.036)$. In comparison to global MAF from the ClinVar database (0.280), MAF findings for IVS2-16G>C in this study were noted higher but lower when compared to the 1000 Genomes Project $(0.720){ }^{22}$. The untranslated region (UTR) is the sequence in the 3' region of a gene but not translated during protein synthesis and contains regulatory element for the gene expression ${ }^{23}$. A variant in the $3^{\prime} U T R$ of the $H B B$ gene, which is assigned as $3^{\prime} U T R+314 G>A$ was found to have a significant susceptibility effect towards beta-thalassemia with the odds ratio $2.013(p=0.004)$. The MAFs were found to be 0.092 in the case group and 0.048 in the control group. However, we noted that there was very limited report of this variant in the literature for further comparison. Overall, we noticed that the MAF for the three significant variants in this study were still-within the range of global MAF from other studies reported in the ClinVar database [21, 22]. The different MAF value could be varied across diverse ethnic or population as well as study sample size ${ }^{24}$.

In an attempt to further evaluate the role of $H B B$ haplotypes in beta-thalassemia in Malaysia, haplotype analysis revealed several susceptible and protective haplotypes 25. The potential applications of haplotype-tagged SNPs have been widely described in the literature. Fields of application include, for example, disease association and pharmacogenetic studies ${ }^{26}$. In this study, we identified seven different haplotypes using the five intragenic HBB SNPs. A comparable finding was reported by Bilgen et al (2011) for the haplotype analysis in the Turkish population. Likewise, the authors have also reported that SNP based haplotyping using five intragenic SNPs has 
successfully established the beta globin gene mutation related haplotypes ${ }^{19}$. In the earlier studies done by Fuchareon et al (2001) and Sanguansermri et al (2004) also have reported association of certain haplotype pattern with $\mathrm{HbE}$ and common betathalassemia mutation respectively by using PCR-RFLP method. To the best of our knowledge, no study was done so far to evaluate the important role of intragenic $H B B$ SNPs in thalassemia syndrome in Southeast Asian region. In this study, we identified six significant haplotypes of which, have important role for beta-thalassemia. Noteworthy, individuals with haplotype that consists of all major alleles from our assigned $H B B$ polymorphisms (1-1-1-1-1) might have a higher risk in developing betathalassemia. However, if the minor allele from IVS2-666C $>$ T is substituted, the effect becomes a protective effect. This allele transition might reveal the protective role from the minor allele of IVS2-666C >T. The same effect is reflected in IVS2-16G>C. However, the protective effect from the minor allele of IVS2-16G>C was not strong enough to confer susceptibility for this haplotype.

Interesting to note that the combination of both minor alleles from IVS2-666C > T and 3'UTR +233G>C with other dominant alleles projected higher protection, which elucidates the same protective role from $3^{\prime} \mathrm{UTR}+233 \mathrm{G}>\mathrm{C}$. These synergist effects provide a better outcome for individuals with this haplotype 1-1-2-2-1. The same synergist effect was also observed for haplotype 2-2-1-1-1, which revealed the protective role from IVS2-74T>G and IVS2-16G>C. Likewise, the allele substitution for 3'UTR +314G>A in haplotype 1-1-2-1-2 dropped the protective effect from haplotype 1-1-2-1-1. The susceptible effect might explain this from a minor allele of 3 'UTR $+314 \mathrm{G}>\mathrm{A}$. This haplotype-based association analysis was carried out to provide a prediction of the predisposing effect and reveal the severity and possible prognosis 
using haplotype-tagged SNPs of HBB gene for beta-thalassemia. Thus, this model could be further developed for the improvement of clinical management of betathalassemia in Malaysia mainly based on the personalized haplotype profile.

In conclusion, the presented study the first study on intragenic polymorphic markers of the beta-globin gene involving the Malaysian population. Identification of susceptible and protective haplotype markers that conferred the significant association with beta-thalassemia in Malaysia can be further refined following the multi-ethnic background of the Malaysian population. The association data on a single genotype and haplotype might disclose the effect of $H B B$ polymorphisms in beta-thalassemia that might provide an impact in the understanding of beta-thalassemia propensity. This study can be ascertained by larger sample size, and stratification by ethnicity should be deliberated since Malaysia is inhibited by various ethnicity.

\section{MATERIALS \& METHODS}

\section{Study population}

This cross-sectional study was conducted among the referral case for DNA analysis of thalassemia syndromes in the Institute for Medical Research (IMR), Kuala Lumpur. The study protocol was approved by the Medical Research Ethics Committee [MREC; NMRR-18-3977-43849 (IIR)] and UniSZA Human Research Ethical Committee [UniSZA/UHREC/2020/170]. Informed consent was obtained from each case prior blood collection was done. Protocol of this study was in accordance with the Declaration of Helsinki. A total of 543 (294 controls \& 249 cases) archived cases from the year 2011 until 2014 were reviewed for this study. Only cases with valid Malaysian 
identity card numbers were included in this study. Cases with no sequencing results and no valid Malaysian identity card numbers were excluded from this study. These cases were molecularly ascertained via Sanger sequencing using $3730 \mathrm{XL}$ DNA Analyser (Applied Biosystem, Foster City, CA, USA) for the presence of $H B B$ gene variation. Samples with heterozygous or compound heterozygous or homozygous state of $H B B$ gene mutations were grouped as cases. Whilst controls were the samples without the known beta-globin gene mutation.

\section{SNP Genotyping}

Genomic DNA was extracted from peripheral blood using a commercial DNA extraction kit (QIAGEN, Germany). The detection of the genotype for IVS2-74T>G (HBB:c.315+74T>G), IVS2-16G>C (HBB:c.315+16G>C), IVS2-666C>T (HBB:c.316185C >T), 3'UTR +233G>C (HBB:C. ${ }^{* 233 G>C)}$ and 3 'UTR +314G>A (HBB:C. $\left.{ }^{*} 314 G>A\right)$ polymorphic site in the $H B B$ gene was performed using a direct DNA sequencing technique in which the cycle sequencing used the BigDye® Terminator v3.1 cycle sequencing kit. Sequence analysis was performed on CLC Main Workbench 6 version 6.6.1 software (CLC Bio, Denmark).

\section{Bioinformatics analysis}

The SHEsis Online software (http://analysis.bio-x.cn/myAnalysis.php) was employed to assess the SNPs and haplotype association in which allelic and genotypic distribution were compared between case and control groups ${ }^{18}$. The odds ratios (ORs) value with a $95 \%$ confidence interval $(95 \% \mathrm{Cl})$ in which a $p$-value of 0.05 was considered as significant. 
Evidence of new intragenic $H B B$ haplotypes of beta-thalassemia

Page 14 of 17

\section{REFERENCES}

1. Old J. Hemoglobinopathies and Thalassemias. Academic Press; :1-44. https://www.sciencedirect.com/science/article/pii/B9780123838346000756?via \%3Dihub. Accessed September 5, 2019.

2. Williams TN, Weatherall DJ. World distribution, population genetics, and health burden of the hemoglobinopathies. Cold Spring Harb Perspect Med. 2012;2(9):a011692. doi:10.1101/cshperspect.a011692

3. Sanctis $\vee$ De, Kattamis $C$, Canatan $D$, et al. $\beta$-thalassemia distribution in the old world: An ancient disease seen from a historical standpoint. Mediterr J Hematol Infect Dis. 2017. doi:10.4084/mjhid.2017.018

4. Greene DN, Vaugn CP, Crews BO, Agarwal AM. Advances in detection of hemoglobinopathies. Clin Chim Acta. 2014. doi:10.1016/j.cca.2014.10.006

5. Modell B, Darlison M. Global Epidemiology of Haemoglobin Disorders and Derived Service Indicators. Vol 86. World Health Organization; 2008:480-487. doi:10.2471/BLT.06.036673

6. Mohd Ibrahim, H.Malaysian Thalassemia Registry Report 2018. 1st ed. Medical Development Division, Ministry of Health M. Malaysian Thalassaemia Registry. Vol 19.; 2019.

7. Tan J a M a, George E, Tan KL, et al. Molecular defects in the beta-globin gene identified in different ethnic groups/populations during prenatal diagnosis for beta-thalassemia: a Malaysian experience. Clin Exp Med. 2004;4(3):142-147. doi:10.1007/s10238-004-0048-x

8. Rosline H, Ahmed SA, Al-Joudi FS, Rapiaah M, Naing NN, Adam NAM. Thalassemia among blood donors at the Hospital Universiti Sains Malaysia. Southeast Asian J Trop Med Public Health. 2006;37(3):549-552. 
http://www.ncbi.nlm.nih.gov/pubmed/17120978. Accessed August 22, 2019.

9. Tan JAMAA, Chin SS, Ong GB, et al. Transfusion-dependent Thalassemia in northern Sarawak: A molecular study to identify different genotypes in the multiethnic groups and the importance of genomic sequencing in unstudied populations. Public Health Genomics. 2015;18(1). doi:10.1159/000368342

10. Teh LK, George E, Lai MI, Tan JAMA, Wong L, Ismail P. Molecular basis of transfusion dependent beta-thalassemia major patients in Sabah. J Hum Genet. 2014;59(3):119-123. doi:10.1038/jhg.2013.131

11. Mohd Yatim NF, Abd. Rahim M, Menon K, et al. Molecular characterization of a- and $\beta$-thalassaemia among Malay patients. Int J Mol Sci. 2014. doi:10.3390/ijms15058835

12. HBB Gene - GeneCards | HBB Protein | HBB Antibody. https://www.genecards.org/cgi-bin/carddisp.pl?gene=HBB. Accessed June 8, 2020.

13. Taghavifar F, Hamid M, Shariati G. Gene expression in blood from an individual with $\beta$-thalassemia: An RNA sequence analysis. Mol Genet Genomic Med. 2019;7(7):e740. doi:10.1002/mgg3.740

14. Brancaleoni V, Di Pierro E, Motta I, Cappellini MD. Laboratory diagnosis of thalassemia. Int J Lab Hematol. 2016;38. doi:10.1111/ijlh.12527

15. Taib WRW, Ahmad N, Ali E, Muda TFMT, Jusoh NM, Rebuan HMA. Suggestive evidence of protective haplotype within TGF-B1 gene region in breast density utilizing fine mapping analysis. Meta Gene. 2020. doi:10.1016/j.mgene.2019.100631

16. Tan J-AMAAMA, Lee P-CC, Wee Y-CC, et al. High prevalence of alpha- and beta-thalassemia in the kadazandusuns in east Malaysia: Challenges in 
providing effective health care for an indigenous group. J Biomed Biotechnol. 2010;2010. doi:10.1155/2010/706872

17. Elizabeth G, Ann MTJA. Genotype-phenotype diversity of beta-thalassemia in Malaysia: Treatment options and emerging therapies. Med J Malaysia. 2010.

18. Yong Y, He L, Shi Yy, He L. SHEsis, a powerful software platform for analyses of linkage disequilibrium, haplotype construction, and genetic association at polymorphism loci. Cell Res. 2005;15:97. https://doi.org/10.1038/sj.cr.7310101.

19. Bilgen T, Arikan Y, Canatan D, Yeşilipek A, Keser I. The association between intragenic SNP haplotypes and mutations of the beta globin gene in a Turkish population. Blood Cells, Mol Dis. 2011. doi:10.1016/j.bcmd.2011.01.004

20. Akhavan-Niaki H, Seresti SS, Asghari B, Banihashemi A. IVSII-666 of human beta-globin gene: A polymorphic marker linked to codon 8(-AA) mutation. Genet Test Mol Biomarkers. 2011. doi:10.1089/gtmb.2010.0242

21. National Center for Biotechnology Information. VCV000036316.3 - ClinVar NCBI. https://www.ncbi.nlm.nih.gov/clinvar/variation/36316/\#id_first. Accessed June 14, 2020.

22. National Center for Biotechnology Information. VCV000256345.3 - ClinVar NCBI. https://www.ncbi.nlm.nih.gov/clinvar/variation/256345/. Accessed June $11,2020$.

23. Barrett LW, Fletcher S, Wilton SD. Untranslated Gene Regions and Other Noncoding Elements. In: ; 2013. doi:10.1007/978-3-0348-0679-4_1

24. Shamsuddin AA, Ahmad A, Wan Taib WR. Haplotype analysis of leptin gene polymorphisms in obesity among Malays in Terengganu, Malaysia population. Med J Malaysia. 2018.

25. Wan Taib WR. Study of Association of TGF-B1 Polymorphism with Breast 
Evidence of new intragenic $H B B$ haplotypes of beta-thalassemia

Page 17 of 17

Density in a Tertiary Medical Center of Malaysia. Med Health. 2017. doi:10.17576/mh.2017.1202.15

26. Ouyang C, Krontiris TG. Identification and functional significance of SNPs underlying conserved haplotype frameworks across ethnic populations. Pharmacogenet Genomics. 2006. doi:10.1097/01.fpc.0000220569.82842.9b

\section{ACKNOWLEDGEMENTS}

The authors would like to thank IMR and MTR for their cooperation and participation in this study. We also would like to thank the Director General of Health, Malaysia, for his permission to publish this article.

\section{AUTHOR CONTRIBUTIONS}

N.A. and W.W.T. wrote the main manuscript text. N.A., N.K., and W.W.T. run the investigation, revised the manuscript for important intellectual content. N.A, N.K., W.W.T., H.A.N.A., W.N.W.A.J., E.E. and H.I. contributed to all aspects of the investigation, including methodological design, data collection and analysis, interpretation of the results. All authors reviewed and revised the manuscript.

\section{DECLARATION OF INTEREST}

The authors report no conflict of interest. The authors alone are responsible for the content and writing of this article.

\section{END OF THIS ARTICLE}

